\title{
Enhancement in Selectivity of Nonenzymatic Glucose Sensors Based on Mesoporous Platinum by A.C. Impedance
}

\author{
Sejin Park and Hankil Boo"** \\ Basic Science Research Institute, Sungshin Women's University, 249-1 Dongsun-dong, Seongbuk-gu, Seoul 136-742, Korea \\ 'The Cooperative Center for Nano-Bio Applied Technology, Sungshin Women's University, 249-1 \\ Dongsun-dong, Seongbuk-gu, Seoul 136-742, Korea
}

(Received June 27, 2008 : Accepted July 16, 2008)

\begin{abstract}
Improvement of the selectivity of nonenzymatic glucose based on mesoporous platinum $\left(\mathrm{H}_{1}-\mathrm{ePt}\right)$ by using A.C. impedance is reported. The idea of the present work is based on the novel effect of the mesoporous electrode that the apparent exchange current due to glucose oxidation remarkably grows although the reaction kinetics on the surface is still sluggish. It is expected that the enlarged apparent exchange current on the mesoporous electrode can raise the sensitivity of admittance in A.C. impedance to glucose concentration. At a low frequency, A.C. impedance could become more powerful. The admittance at $0.01 \mathrm{~Hz}$ is even more sensitive to glucose than to ascorbic acid while amperometry exhibits the inverse order of sensitivity. This is the unique behavior that is neither observed by A.C. impedance on flat platinum electrode nor obtained by amperometry. The study shows how the combination of A.C. impedance and nano-structured surface can be applied to the detection of sluggish reaction such as electrochemical oxidation of glucose.
\end{abstract}

Keywords : Impedance,Mesoporous platinum, Nonenzymatic glucose sensor, Nano-structured surface.

\section{Introduction}

Many studies of nonenzymatic glucose detection have been performed to avoid the drawbacks of enzymatic glucose sensors, their lack of stability of enzymes. Basic studies of the electrochemical oxidation of glucose on the electrodes were reported, ${ }^{1-7)}$ and amperometric measurements of glucose were attempted. ${ }^{8,9)}$ Another approaches were also taken, for example, electrochemical techniques combined with chemical receptor for glucose. ${ }^{10-13)}$ Most of the previous studies of the amperometric detection by direct electrochemical oxidation of glucose did not overcome the problems of low selectivity and sensitivity, poisoning by chloride ion and toxicity of heavy metal atoms modifying the electrode.

But, our previous report on the nonenzymatic glucose detection using mesoporous platinum $\left(\mathrm{H}_{1}-\mathrm{ePt}\right)$ showed astonishing improvement in terms of sensitivity, selectivity, and poisoning by chloride ion. ${ }^{9)}$ We showed

*E-mail: sizyphe@sungshin.ac.kr in that report that bare Pt with nanoscopic roughness could be successfully applied to amperometric detection of glucose even if biological concentration of ascorbic acid (AA) and 4-acetamidophenol (AP) presented as interferences. The key idea of that study is based on the fact that the roughness of mesoporous electrodes is even smaller than the scale of the chronoamperometric diffusion field in most cases. Since the diffusion layers extend several micrometers away from the electrode surface in milliseconds, reactants inside the mesopores $(2 \sim 50 \mathrm{~nm}$ in diameter $)^{14)}$ are depleted in diffusion-controlled electrochemical systems as illustrated in Fig. 1(A). As a result, the faradaic currents of rapidly oxidizable and/or reducible reactants are proportional to the apparent geometric area of the electrode, regardless of its mesoporous roughness. ${ }^{15)}$ On the other hand, faradaic currents associated with kinetic-controlled electrochemical events are sensitive to the nanoscopic surface area of the electrode, rather than to its geometric area (Fig. 1(B)). Deeper and denser mesopores on an electrode surface generate larger roughness factors, 


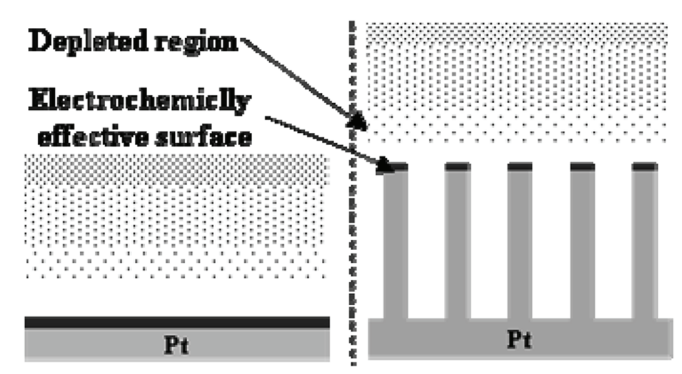

(A) Difinsion controlled electron transibr (es. As or AP)

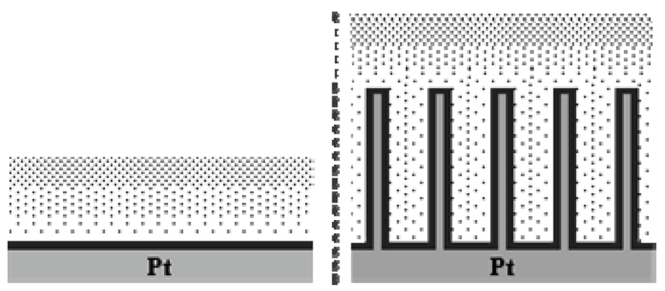

(B) Kinetic controlled electron transfer (e.B. Blucose)

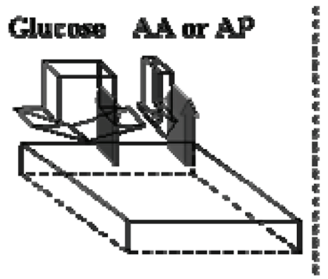

(C)

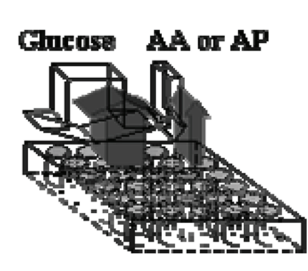

Mescpareus Pt
Fig. 1. Schematic representation of the effect of pore structure on electrochemical reactions.

that is, the ratio of nanoscopic surface area to the geometric surface area. The fabrication method and the properties of $\mathrm{H}_{1}$-ePt films with cylindrical hexagonally arrayed pores (pore diameter, $2.5 \mathrm{~nm}$; pore-pore distance $5.0 \mathrm{~nm}$ ) have been previously reported upon. ${ }^{9,14,16-29)}$ Highly enlarged electrode areas, in nanoscale terms, boost the faradaic currents of the sluggish reaction exclusively as depicted in Fig. 1(C).

The study of the amperometric detection of glucose on $\mathrm{H}_{1}$-ePt shows one methodology how the sensitivity for a kinetic-controlled reaction vs. diffusion-controlled one can be enhanced on bare electrode without any chemical or enzymatic treatments. Another methodology for the exclusively selective enhancement of kineticcontrolled reaction is the use of A.C. impedance.

A series of reports of Hall et al. ${ }^{30-33)}$ are the only examples that A.C. impedance technique was used to accomplish nonenzymatic and selective detection. They demonstrated a selective detection for hydrogen peroxide in the presence of ascorbic acid as interfering species by measuring A.C. impedance. The selective detection was successful under specific measurement condition, that is, (1) dc-potential range where the oxidation of ascorbic acid occurs in a diffusion-controlled manner whereas that of hydrogen peroxide occurs in a kinetic-controlled manner and (2) low A.C. frequencies, where the contribution of the double layer was not significant. Intuitively, if the electrochemical reaction becomes more diffusion limited, the magnitude of the acresponse diminishes, whereas an oscillation in the given potential still allows an appreciable oscillation of current in the kinetic-controlled system. Thus, the A.C. signal of hydrogen peroxide was larger than ascorbic acid when it was measured at dc-potential where the electrochemical oxidation of hydrogen peroxide was under kinetic control and that of ascorbic acid was under diffusion control. This is the first trial to tune the A.C. signal for greater sensitivity to the kinetically less-favored species, which was not available by traditional amperometry. ${ }^{32}$ )

As noted above, although our previous work on the amperometric detection of glucose using mesoporous $\mathrm{Pt}$ showed good selectivity in condition of physiological concentration of ascorbic acid and acetamidophenol, it is more desirable to achieve higher selectivity. Because the electrochemical oxidation of glucose is diffusion-controlled, a similar situation met by Hall et al. is expected to be valid for the mixture of glucose and ascorbic acid. Now, we expect that adopting A.C. impedance technique will be an effective approach to enhance the nonenzymatic selectivity of mesoporous Pt electrode.

\section{Electrochemical Experiments}

\section{Reagents}

Octaethylene glycol monohexadecyl ether $\mathrm{C}_{16} \mathrm{EO}_{8}$ (Fluka), hydrogen hexachloroplatinate hydrate (Aldrich), sulfuric acid, D-(+)-glucose (Sigma), L-ascorbic acid (Aldrich), and 4-acetamidophenol (Sigma) were used without purification.

\section{Instruments}

Electrochemical experiments were performed 
with an electrochemical analyzer (Model CH660, $\mathrm{CH}$ Instruments Inc., Austin, TX 78733). $\mathrm{Ag} / \mathrm{AgCl}$ (3 M $\mathrm{KCl})$ and platinum foil $(5 \mathrm{~cm} \times 5 \mathrm{~cm})$ were used as a reference and counter electrode, respectively. A platinum rod electrode $\left(0.02 \mathrm{~cm}^{2}\right)$ was used as a substrate electrode for $\mathrm{H}_{1}$-ePt film.

\section{Preparation of liquid crystal}

$\mathrm{C}_{16} \mathrm{EO}_{8}(0.42 \mathrm{~g})$, distilled water $(0.29 \mathrm{~g})$, and hydrogen hexachloroplatinate hydrate $(0.29 \mathrm{~g})$ were mixed, and the temperature was raised to $80^{\circ} \mathrm{C}$ until the mixture became transparent and homogeneous. Electrodes were inserted into the homogeneous mixture, and the temperature was lowered to room temperature $\left(\sim 23 \sim 26^{\circ} \mathrm{C}\right)$. At this stage, the mixture became a highly viscous liquid crystalline phase.

\section{Electrodeposition of $\mathbf{H}_{1}-\mathbf{e P t}$}

Platinum deposition was carried out on a polished platinum rod electrode at constant potential $(-0.06 \mathrm{~V}$ vs. $\mathrm{Ag} / \mathrm{AgCl})$. The resulting $\mathrm{H}_{1}$-ePt electrode was placed in distilled water for $1 \mathrm{hr}$ to extract $\mathrm{C}_{16} \mathrm{EO}_{8}$. After the extraction was repeated 3 4 times, electrochemical cleaning was performed using a cycling potential between +1.0 and $-0.45 \mathrm{~V}$ versus $\mathrm{Ag} / \mathrm{AgCl}$ in $0.5 \mathrm{~N}$ sulfuric acid until reproducibly identical cyclic voltammograms were obtained.

\section{Area measurements and electrochemical cleaning}

All the electrochemical measurements were done in 3-electrode system. The surface area of Pt was determined by measuring the area under the hydrogen desorption peak of cyclic voltammogram in $0.5 \mathrm{~N}$ sulfuric acid solution. Electrochemical cleaning of the platinum electrode was performed before each measurement by applying potential step from $-0.2 \mathrm{~V}$ $(5 \mathrm{sec})$ to $1.2 \mathrm{~V}(20 \mathrm{Sec}) \mathrm{vs} . \mathrm{Ag} / \mathrm{AgCl}$ in $0.5 \mathrm{~N}$ sulfuric and transferring the platinum electrode to $0.5 \mathrm{~N}$ $\mathrm{KOH}$ to dissolve out the adsorbed oxidative product. After area of the electrode was measured in sulfuric acid solution by cyclic voltammetry, and then it was used for the electrochemical measurement.

\section{Electrochemical measurements}

Evaluation of $\mathrm{H}_{1}$-ePt electrode as a glucose sensor was performed in aerated $0.1 \mathrm{M}$ phosphate buffered saline (PBS) solution containing $0.15 \mathrm{M} \mathrm{NaCl}$, and $\mathrm{NaN}_{3}$ was not used because it blocks the oxidation of glucose. The dc-amperometric curves were obtained in a quiescent solution a few seconds after stopping the stirring that was required to mix the materials added (i.e., glucose, AA, and AP). Current changes 100 seconds after adding glucose to specific were treated as specific responses to the glucose in the solution. The signals for AA and AP were measured 100 seconds later.

Impedance measurements were carried out at dcpotential of $0.4 \mathrm{~V}$ vs. $\mathrm{Ag} / \mathrm{AgCl}$ with A.C. amplitude of $10 \mathrm{mV}$. The measurements were begun after 120 seconds of pre-equilibrium time from $100 \mathrm{kHz}$ to $0.01 \mathrm{~Hz}$.

\section{Results and Discussion}

The amperometric signal of ascorbic acid (AA) is ca. 2.8 times larger than that of glucose of the same concentration, $6 \mathrm{mM}$ on $\mathrm{H}_{1}$-ePt (Fig. 2). According to the result of previous report, ${ }^{9} \mathrm{Pt}-\mathrm{s}$ (roughness factor, 2.6) shows signals of $12.3 \mu \mathrm{Acm}^{-2}$ for $0.1 \mathrm{mM} \mathrm{AA}$ and $0.138 \mu \mathrm{Acm}^{-2}$ for $6.0 \mathrm{mM}$ glucose, and the sensitivity for $\mathrm{AA}$ is ca. 5,000 times higher than that of glucose of the same concentration. However, the amperometric signal from ascorbic acid is still larger than that from glucose, and so more improvement in selectivity is required.

In the present study, we combine A.C. amperometric measurement with mesoporous surface of $\mathrm{H}_{1}-\mathrm{ePt}$ to improve the selectivity for glucose. The dc-potential on which A.C. perturbation would be added was determined to be $+0.4 \mathrm{~V}$ vs. $\mathrm{Ag} / \mathrm{AgCl}$. The amperometric

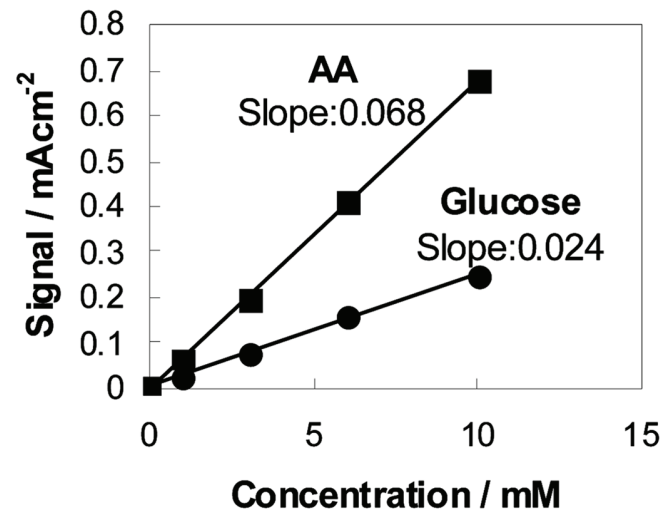

Fig. 2. Typical dc-amperometric signals measured on $\mathrm{H}_{1}-\mathrm{ePt}$ (roughness factor: 225) in PBS solution containing glucose (circle) and $\mathrm{AA}$ (square) of the same concentration (6 mM). 
determination was also performed at the same potential. Possible reactions at the potential are platinum oxide formation, oxidation of ascorbic acid, and oxidation of glucose.

In PBS solution, Pt surface undergoes oxide formation whether the glucose or AA exists or not. Among numerous reports by many authors, ${ }^{1-7)}$ there is a common feature on the mechanism for the oxide formation. Briefly, one-electron (per one Pt atom) transfer reaction occurs below the specific potential (i.e., $1.1 \mathrm{~V}$ vs. RHE for $0.5 \mathrm{M} \mathrm{H}_{2} \mathrm{SO}_{4}$ solution) and then another one-electron transfer reaction occurs coupled with place change reaction of platinum and oxygen at higher potential. Ascorbic acid is oxidized on platinum in the potential range where platinum oxide is formed. The mechanism of ascorbic acid oxidation was proposed as the reversible adsorption on platinum covering ca. $68 \%$ of the hydrogen adsorption sites, and then oxidation to dehydroascorbic acid. ${ }^{34-36)}$ Glucose undergoes electrochemical oxidation on Pt in three potential ranges; hydrogen adsorption, double layer, and oxide formation region.

From cyclic voltammogram of previous report, ${ }^{9)}$ it was confirmed that platinum oxide formation and glucose oxidation in platinum oxide region begin near $0.4 \mathrm{~V}$ vs. $\mathrm{Ag} / \mathrm{AgCl}$. However, the oxidation current peak for $\mathrm{AA}$ is shown near $0.13 \mathrm{~V}$ vs. $\mathrm{Ag} / \mathrm{AgCl}$, and diffusion current was observed at $0.4 \mathrm{~V}$. Sum-

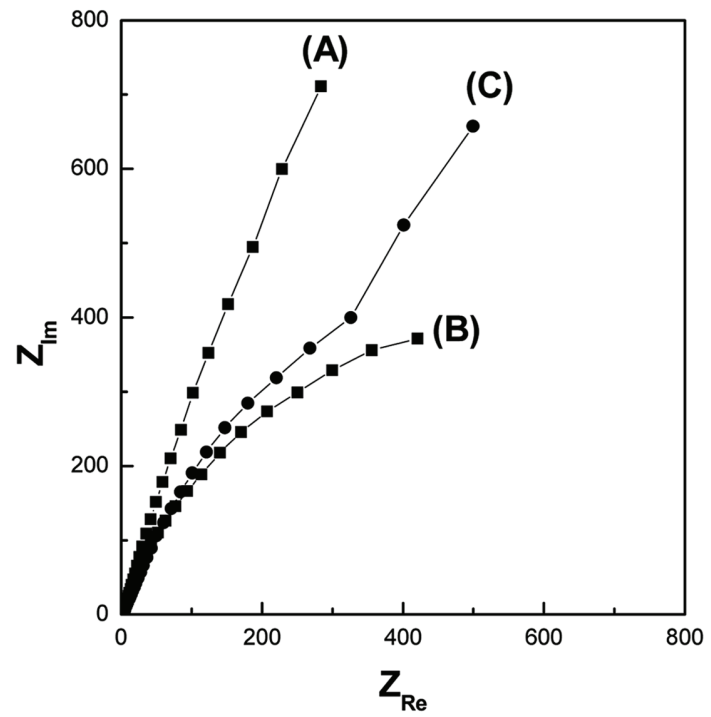

Fig. 3. Nyquist plots of $\mathrm{H}_{1}-\mathrm{ePt}$ in (A) PBS solution, (B) glucose, and (C) AA solution. marizing the situation of $\mathrm{H}_{1}-\mathrm{ePt}$ at $0.4 \mathrm{~V}$ vs. $\mathrm{Ag} /$ $\mathrm{AgCl}$, (1) faradaic current flows due to formation of platinum oxide within mono-layer, diffusion-controlled oxidation of AA, and kinetic-controlled oxidation of glucose (2) and faradaic current due to platinum oxide formation always exists as background, but its magnitude should be very small because the oxidation rarely occurs beyond mono-layer.

The measurements were done in three kinds of solutions, PBS blank solution and PBS solutions containing $6 \mathrm{mM}$ glucose or $6 \mathrm{mM}$ ascorbic acid (AA). Nyquist plots in Fig. 3 show the impedance spectra measured with $\mathrm{H}_{1}$-ePt in PBS solution (A) and then with (B) glucose or (C) AA added to PBS solution. Because AA is oxidized much faster than glucose at the electrode and the concentration of AA of the electrode surface is depleted, the oxidation of AA is mass-transfer controlled and Warburg impedance become important at low frequencies. On the other hand, Warburg impedance of glucose oxidation is neglected at given frequencies due to the slow kinetics of glucose oxidation.

Table 1 shows the admittance values at $0.012 \mathrm{~Hz}$ at each solution. Clear amperometric response was observed for glucose at A.C. frequency of $0.012 \mathrm{~Hz}$. The addition of glucose increases the admittance by 0.5 , due to the decrease of charge transfer resistance. However, the addition of ascorbic acid decreases the admittance by 0.1 . The presence of ascorbic acid affects on the dc-amperometric signal by ca. 2.8 times more than glucose, but affects on the A.C. amperometric signal only by 0.20 times. It is clear that the signal for glucose becomes much larger than that of ascorbic acid, when A.C. impedance gets combined with $\mathrm{H}_{1}-\mathrm{ePt}$ instead of amperometry, although the improvement ratio

Table 1. Results of A.C. impedance and amperometry performed with $\mathrm{H}_{1}-\mathrm{ePt}$

\begin{tabular}{cccc}
\hline & $\mathrm{Y} / \Omega^{-1}$ at $0.012 \mathrm{~Hz}^{a}$ & $\Delta^{b}$ & $\mathrm{I} / \mathrm{mAcm}^{-2} \mathrm{~mol}^{-1, c}$ \\
\hline PBS & 1.3 & - & - \\
Glucose & 1.8 & 0.5 & 0.024 \\
AA & 1.2 & -0.1 & 0.068 \\
\hline Ratio $^{d}$ & & 0.20 & 2.8
\end{tabular}

$a_{\text {admittance measured by A.C. impedance with } \mathrm{H}_{1}-\mathrm{ePt}}$ (roughness factor: 149).

${ }^{b}$ difference of admittance from that of PBS.

${ }^{c}$ current density measured by dc-amperometry signal measured with $\mathrm{H}_{1}$-ePt (roughness factor, 225, from slope in Fig. 2). ${ }^{d}$ ratios of measured values for AA to those for glucose. 


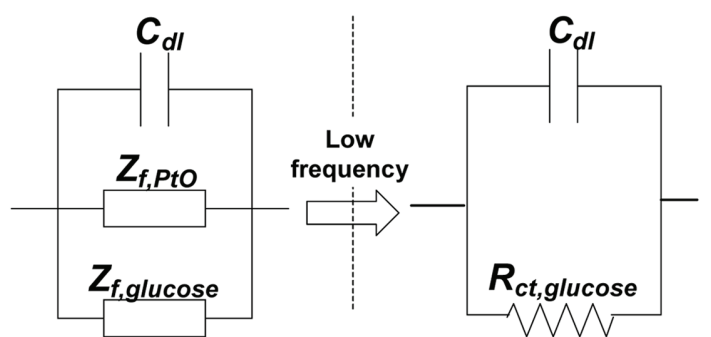

(A)

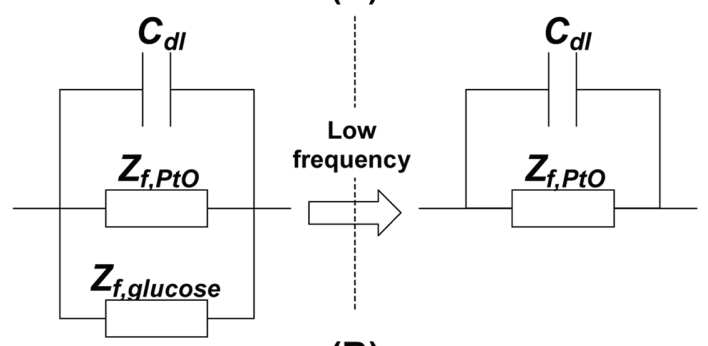

(B)

Fig. 4. Schematic presentation of the approximated equivalent circuits for (A) glucose and (B) AA at low frequency.

may depend on the roughness factor and other measurement conditions, e.g. dc-potential, $\mathrm{pH}$, etc.

The reason why the A.C. impedance signal for glucose is larger than AA can be explained in the same ways how Hall et al. explained higher admittance for hydrogen peroxide than for AA. ${ }^{37-41)}$ Analogously to their principle, the admittance for AA oxidation (a diffusion-controlled reaction) is much smaller than that of oxidation of glucose (a kinetic-

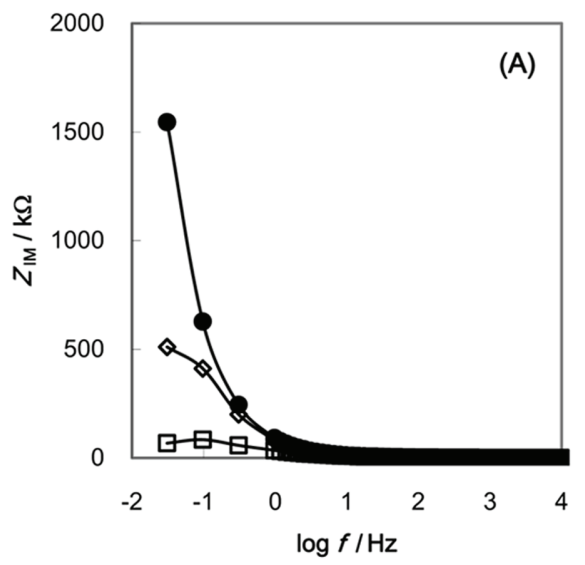

controlled reaction), at sufficiently low frequency.

The equivalent circuits for the platinum surface in the PBS solution containing glucose or AA are presented in Fig. 4. Because the faradaic current path for platinum oxide formation exists always, the impedance element for the path $\left(Z_{f, P t O}\right)$ should be placed in parallel with the other faradaic impedance elements for the electrochemical oxidation of glucose $\left(Z_{f, \text { glucose }}\right)$ or AA $\left(Z_{f, A A}\right)$. In PBS solution containing glucose (Fig. 4(A)), $Z_{f, \text { glucose }}$ is approximated to charge transfer resistance $\left(R_{c t, \text { glucose }}\right)$, because the oxidation of glucose is kinetic-controlled. Moreover, $R_{c t, \text { glucose }}$ is smaller than $Z_{f, P t O}$, and so the whole equivalent circuit can be represented by parallel combination of double layer capacitance and $R_{c t, \text { glucose }}$. In the case of AA, $Z_{f, A A}$ can be approximated to Warburg impedance $\left(Z_{W, A A}\right)$, because the electrochemical oxidation of $\mathrm{AA}$ is diffusion-controlled. Because the magnitude of $Z_{w}$ is proportional to $\omega^{-1 / 2}$ as equation 1 , the impedance of ascorbic acid becomes infinite as frequency decreases.

$$
\left|Z_{W}\right|=\left(\frac{2}{\omega}\right)^{1 / 2} \sigma
$$

Thus, it is expected that the faradaic impedance resulted from the oxidation of ascorbic acid has very large value in low frequency range. If the frequency is low enough for the $Z_{f, A A}$ to be much larger than $Z_{f, P t O}$, the total impedance may be determined by the parallel combination of $C_{d l}$ and $Z_{f, P t O}$.

Actually, the Nyquist plot of AA in Fig. 3 show similar shape and values with PBS in low frequency

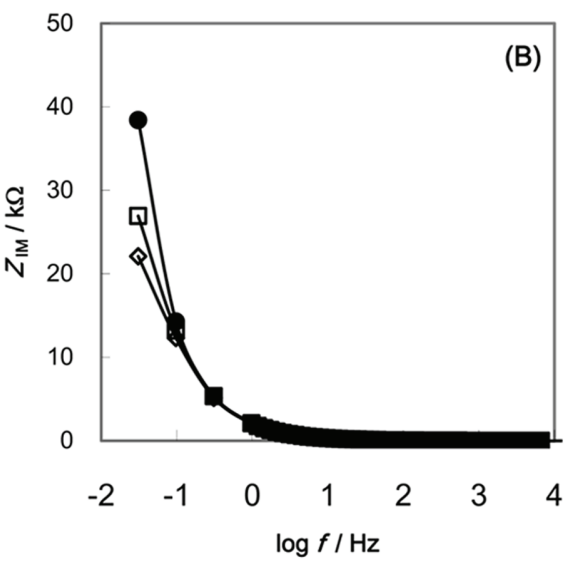

Fig. 5. Plots of $Z_{\mathrm{IM}}$ vs. frequency measured with (A) flat $\mathrm{Pt}$ (r.f., 120) and (B) $\mathrm{L}_{2}$-ePt (r.f., 120) at dc potential of $0.4 \mathrm{~V}$ vs. $\mathrm{Ag} / \mathrm{AgCl}$ and A.C. amplitude of $10 \mathrm{mV}$. PBS blank solution (solid circle), and PBS solution containing 6 mM AA (open square) or $6 \mathrm{mM}$ glucose (open diamond). 
range. For glucose, Warburg impedance does not appear in the measured frequency range, and the impedance plot falls into the part of semicircle with diameter corresponding to the value of charge transfer resistance $\left(R_{\text {ct,glucose }}\right)$, which decreases as the concentration of glucose increases.

As a result, in sufficiently low frequency range, $Z_{f, A A}$ does not significantly affect on the whole impedance, whereas $Z_{f, \text { glucose }}$ predominates the whole impedance. Thus, addition of AA does scarcely changes the A.C. impedance, but the increase of glucose concentration results in the decrease of A.C. impedance (increase of A.C. admittance).

It is noticeable that the impedance change owing to glucose is obvious on mesoporous electrode surface, whereas the impedance change is negligible on flat surface (solid circle in Fig. 5(A) and (B)). On Pt-s, the oxidation of glucose is too slow to result in the difference in admittances. Furthermore, Pt-s responses very sensitively to the presence of AA. Thus, it is the use of mesoporous structured surface that makes the selectivity improvement by A.C. impedance method possible, in terms of glucose.

Although previous works by Hall et al. showed successful results in discrimination of hydrogen peroxide from ascorbic acid on flat glassy carbon electrode, it was a demonstration example that can be applied to the redox reactions whose rate is high enough to result in the increase of admittance even on a flat electrode surface. The reactions with negligibly slow reaction-rate have been out of the application range of A.C. impedance method combined with flat electrode surface. The present study is the first demonstration, in our knowledge, that realizes A.C. impedance detection of very sluggish reaction.

\section{Conclusions}

We accomplished more improvement of nonenzymatic selectivity for glucose of mesoporous platinum $\left(\mathrm{H}_{1}-\mathrm{ePt}\right)$ by using A.C. impedance instead of amperometry. It was possible only on a mesoporous surface, which boosts up the sluggish reaction of glucose. We believe that this study is an important example showing that mesoporous surface makes an analytical method (i.e. A.C. impedance detection of glucose), which has been inapplicable to species with sluggish electron transfer kinetics (i.e. glucose) applicable.

\section{Acknowledgement}

"This work was supported by the Korea Research Foundation Grant funded by Korea Government(MOEHRD)" (KRF-2006-353-C00024)

\section{References}

1. Y. B. Vassilyev, O. A. Khazova, and N. N. Nikolaeva, "kinetics and mechanism of glucose electrooxidation on different electrode-catalysts: Part I. Adsorption and oxidation on platinum" J. Electroanal. Chem., 196, 105 (1985).

2. B. Beden, F. Largeaud, K. B. Kokoh, and C. Lamy, "Fourier transform infrared reflectance spectroscopic investigation of the electrocatalytic oxidation of -glucose: Identification of reactive intermediates and reaction products" Electrochim. Acta, 41, 701 (1996).

3. I. T. Bae, E. Yeager, X. Xing, and C. C. Liu, "In situ infrared studies of glucose oxidation on platinum in an alkaline medium" J. Electroanal. Chem., 309, 131 (1991).

4. M. Sakamoto and K. Takamura, "Catalytic oxidation of biological components on platinum electrodes modified by adsorbed metals: Anodic oxidation of glucose" Bioelectrochem. Bioener, 9, 571 (1982).

5. G. Kokkinidis and N. Xonoglou, "Comparative study of the electrocatalytic influence of underpotential heavy metal adatoms on the anodic oxidation of monosaccharides on Pt in acid solutions" Bioelectrochem. Bioener., 14, 375 (1985).

6. G. Wittstock, A. Strubing, R. Szargan, and G. Werner, "Glucose oxidation at bismuth-modified platinum electrodes" J. Electroanal. Chem., 444, 61 (1998).

7. X. Zhang, K.-Y. Chan, J.-K. You, Z.-G. Lin, and A. C. C. Tseung, "Partial oxidation of glucose by a $\mathrm{PtWO}_{3}$ electrode" J. Electroanal. Chem., 430, 147 (1997).

8. Y. Sun, H. Buck, and T. E. Mallouk, "Combinatorial Discovery of Alloy Electrocatalysts for Amperometric Glucose Sensors" Anal. Chem., 73, 1599 (2001).

9. S. Park, T. D. Chung, and H. C. Kim, "Nonenzymatic glucose detection using mesoporous platinum" Anal. Chem., 75, 3046 (2003).

10. E. Shoji, and M. S. Freund, "Potentiometric Sensors Based on the Inductive Effect on the $\mathrm{pKa}$ of Poly(aniline): A Nonenzymatic Glucose Sensor" J. Am. Chem. Soc., 123, 3383 (2001).

11. E. Shoji and M. S. Freund, "Potentiometric Saccharide Detection Based on the pKa Changes of Poly(aniline boronic acid)" J. Am. Chem. Soc., 124, 12486 (2002).

12. S. Arimori, S. Ushiroda, L. M. Peter, A. T. A. Jenkins, and T. D. James, "A modular electrochemical sensor for saccharides" Chem. Commun., 2368 (2002).

13. S.-J. Choi, B.-G. Choi, and S.-M. Park, "Electrochemical Sensor for Electrochemically Inactive -D(+)-Glucose Using -Cyclodextrin Template Molecules" Anal. Chem., 74, 1998 (2002). 
14. B. Gollas, J. M. Elliott, and P. N. Bartlett, "Electrodeposition and properties of nanostructured platinum films studied by quartz crystal impedance measurements at $10 \mathrm{MHz}$ "Electrochim. Acta, 45, 3711 (2000).

15. A. J. Bard and L. R. Faulkner, "Electrochemical methods: Fundamentals and Applications", Wiley, New York (2001).

16. G. S. Attard, J. C. Glyde, and C. G. Goeltner, "Liquidcrystalline phases as templates for the synthesis of mesoporous silica" Nature, 378, 366 (1995).

17. G. S. Attard, C. G. Goeltner, J. M. Corker, S. Henke, and R. H. Templer, "Liquid-crystal templates for nanostructured metals" Angew. Chem. Int. Ed., 36, 1315 (1997).

18. J. M. Elliott, P. R. Birkin, P. N. Bartlett, and G. S. Attard, "Platinum Microelectrodes with Unique High Surface Areas" Langmuir, 15, 7411 (1999).

19. S. A. G. Evans, J. M. Elliott, L. M. Andrews, P. N. Bartlett, P. J. Doyle, and G. Denuault, "Detection of hydrogen peroxide at mesoporous platinum microelectrodes" Anal. Chem., 74, 1322 (2002).

20. G. S. Attard, M. Edgar, and C. G. Goeltner, "Inorganic nanostructures from lyotropic liquid crystal phases" Acta Mater, 46, 751 (1998).

21. G. S. Attard, P. N. Bartlett, N. R. B. Coleman, J.M. Elliott, and J. R. Owen, "Lyotropic Liquid Crystalline Properties of Nonionic Surfactant/H2O/Hexachloroplatinic Acid Ternary Mixtures Used for the Production of Nanostructured Platinum" Langmuir, 14, 7340 (1998).

22. P. R. Birkin, J. M. Elliott, and Y. E. Watson, "Electrochemical reduction of oxygen on mesoporous platinum microelectrodes" Chem. Commun., 1693 (2000).

23. J. M. Elliott and J. R. Owen, "Electrochemical impedance characterisation of a nanostructured (mesoporous) platinum film" Phys. Chem. Chem. Phys., 2, 5653 (2000).

24. G. S. Attard, P. N. Bartlett, N. R. B. Coleman, J. M. Elliott, J. R. Owen, and J. H. Wang, "Mesoporous platinum films from lyotropic liquid crystalline phases " Science, 278, 838 (1997).

25. J. M. Elliott, G. S. Attard, P. N. Bartlett, N. R. B. Coleman, D. A. S. Merckel, and J. R. Owen, "Nanostructured Platinum (HI-ePt) Films: Effects of Electrodeposition Conditions on Film Properties" Chem. Mater., 11, 3602 (1999).

26. P. N. Bartlett and S. Guerin, "A micromachined calorimetric gas sensor: an application of electrodeposited nanostructured palladium for the detection of combustible gases" Anal. Chem., 75, 126 (2003).

27. J. J. Jiang and A. Kucernak, "Oxygen reduction studies of templated mesoporous platinum catalysts" Electrochemical and Solid State Letters, 3, 559 (2000).

28. J. Jiang and A. Kucernak, "Electrooxidation of small organic molecules on mesoporous on precious metal catalysts I: CO and methanol on platinum" J. Electroanal. Chem., 533, 153 (2002).

29. J. Jiang and A. Kucernak, "Nanostructured platinum as an electrocatalyst for the electrooxidation of formic acid" $J$. Electroanal. Chem., 520, 64 (2002).

30. S. Iyengar, E. A. H. Hall, N. G Skinner, and J. J. Gooding, "Frequency domain selection of the peroxide signal for amperometric biosensors" Electroanalysis, 10, 1089 (1998).

31. S. Iyengar and E. A. H. Hall, "Selective monitoring of the hydrogen peroxide signal in the presence of ascorbic acid. Part II: Preliminary practical realization of applying immittance spectroscopy" Electroanalysis, 13, 517 (2001).

32. S. Iyengar and E. A. H. Hall, "Applying immittance spectroscopy to monitoring hydrogen peroxide in the presence of ascorbic acid. Part I: Theoretical considerations" Electroanalysis, 13, 437 (2001).

33. S. Iyengar and E. A. H. Hall, "Data from overlapping signals at an amperometric electrode using admittance vectors" J. Electroanal. Chem., 521, 61 (2002).

34. K. B. Kokoh, F. Hahn, A. Metayer, and C. Lamy, "FTIR spectroelectrochemical investigation of the electrocatalytic oxidation of ascorbic acid at platinum electrodes in acid medium" Electrochim. Acta, 47, 3965 (2002).

35. M. Brezina, J. Koryta, T. Loucka, D. Marsikova, and J. Pradac, "Adsorption and kinetics of oxidation of ascorbic acid at platinum electrodes" J. Electroanal. Chem., 40, 13 (1972).

36. P. Karabinas and D. Jannakoudakis, "Kinetic parameters and mechanism of the electrochemical oxidation of L-ascorbic acid on platinum electrodes in acid solutions" $J$. Electroanal. Chem., 160, 159 (1984).

37. S. B. Hall, E. A. Khudaish, and A. L. Hart, "Electrochemical oxidation of hydrogen peroxide at platinum electrodes. Part 1. An adsorption-controlled mechanism" Electrochim. Acta, 43, 579 (1998).

38. S. B. Hall, E. A. Khudaish, and A. L. Hart, "Electrochemical oxidation of hydrogen peroxide at platinum electrodes. Part II: effect of potential" Electrochim. Acta, 43, 2015 (1998).

39. S. B. Hall, E. A. Khudaish, and A. L. Hart, "Electrochemical oxidation of hydrogen peroxide at platinum electrodes. Part III: Effect of temperature" Electrochim. Acta, 44, 2455 (1999).

40. S. B. Hall, E. A. Khudaish, and A. L. Hart, "Electrochemical oxidation of hydrogen peroxide at platinum electrodes. Part IV: phosphate buffer dependence" Electrochim. Acta, 44, 4573 (1999).

41. S. B. Hall, E. A. Khudaish, and A. L. Hart, "Electrochemical oxidation of hydrogen peroxide at platinum electrodes. Part V: inhibition by chloride" Electrochim. Acta, 45, 3573 (2000). 